\author{
Д.Н. Суслов ${ }^{1}$, А.В. Матвеева², Н.Н. Рожков ${ }^{2}$, О.А. Герасимова ${ }^{1,3}$, Ф.К. Жеребцов ${ }^{1}$ \\ ${ }^{1}$ ФББУ «Российский научный центр радиологии и хирургических технологий имени академика А.М. Гранова» Министерства \\ здравоохранения Российской Федерации, Санкт-Петербург, РФ \\ 2 ФГБОУ ВО «Санкт-Петербургский государственный университет промышленных технологий и дизайна», Санкт-Петербург, РФ \\ ${ }^{3}$ ФБОУ ВО «Санкт-Петербургский государственный университет», Санкт-Петербург, РФ
}

\title{
ОПТИМИЗАЦИЯ НАБЛЮДЕНИЯ И ЛЕЧЕНИЯ ПАЦИЕНТОВ В «ЛИСТЕ ОЖИДАНИЯ» ПЕЧЕНОЧНОГО ТРАНСПЛАНТАТА. ОПЫТ РЕГИОНАЛЬНОГО ЦЕНТРА
}

Работа лечебных учреждений в условиях внедрения элементов страховой медицины предполагает проверку соблюдения требований и нормативов медико-экономических стандартов (МЭС), или как принято в настоящее время, «тарифа».Учитывая, что в данной статье приводятся результаты внутреннего контроля качества исполнения медико-экономического стандарта «Амбулаторное ведение пациентов в «листе ожидания» трансплантата печени», мы позволили себе использовать название «Медикоэкономический стандарт». Для оценки соблюдения требований и нормативов стандарта была разработана методика, основанная на математической модели [1], нашедшая применение при анализе исполнения МЭС для ряда близких по своему характеру заболеваний. Результаты расчетов выявили статистическую однородность основных показателей, характеризующих выполнение указанных стандартов. Полученные результаты позволили обосновать разработку нового специализированного МЭС: «Амбулаторное ведение больного в листе ожидания печеночного трансплантата», включающего несколько сходных по своей природе заболеваний. Ряд авторов приводят данные о более 30 заболеваниях и состояниях, основным методом коррекции которых служит пересадка печени [2]. При составлении такого стандарта помимо расчетов по методике [1] учитывались требования отраслевых нормативов и собственный опыт ведения пациентов, относящихся к отмеченной категории. Медико-экономический стандарт «Амбулаторное ведение пациентов в «листе ожидания» трансплантата печени» на протяжении трех последних лет применяется для обследования и поддержки пациентов, находящихся в «листе ожидания трансплантата печени» в ФГБУ «Российский научный Центр радио- логии и хирургических технологий». Накопленный опыт применения данного МЭС в клинической практике потребовал выполнить анализ его исполнения и наметить пути совершенствования стандарта.

После включения в «лист ожидания» (ЛО) пациент нуждается в постоянном динамическом наблюдении и симптоматической терапии. Применяемые для этого лабораторные и диагностические услуги в ряде случаев являются высокотехнологическими и высокозатратными [3]. Они не могут быть оказаны в амбулаторных лечебнопрофилактических учреждениях (ЛПУ) по месту жительства пациента. Кроме того, пациенту выполняются специфические исследования, необходимые для составления предварительного плана оперативного вмешательства [4]. Потенциальные реципиенты, ожидающие пересадку печени, и их родственники нуждаются в психотерапевтическом сопровождении [5]. Нам представляется, что проводить подобное наблюдение и лабораторное обследование целесообразно в том Центре, где пациент ожидает оперативное лечение - трансплантацию печени. Это соответствует утвержденному Министерством здравоохранения РФ «Порядку оказания медицинской помощи по профилю «хирургия (трансплантация органов и (или) тканей человека)» (утвержден приказом Министерства здравоохранения РФ от 31 октября 2012 г. N 567н). Кроме того, такая организация ЛО необходима прежде всего в интересах пациента - своевременная диагностика и быстрое начало лечения при возникновении определенных состояний, возмож-

() Д.Н. Суслов, А.В. Матвеева, Н.Н. Рожков,

О.А. Герасимова, Ф.К. Жеребцов, 2018

(c) Университетская Клиника, 2018 
ность динамической оценки состояния пациента по динамике лабораторных показателей - исследования выполняются в одной лаборатории, отсутствует разница в результатах, вызванная погрешностью различных аппаратов и методик выполнения исследований, пациент лишен необходимости поиска ЛПу, где могут быть выполнены необходимые исследования и ожидания выполнения этих исследований. Подобная организация ведения ЛО обеспечивает реализацию рекомендаций Европейского регионального бюро ВО3, которые включают 4 основных компонента: квалификация врача; оптимальность использования ресурсов; низкий риск для пациентов; удовлетворенность пациента оказанной медицинской помощью.

\section{МАТЕРИАЛ И МЕТОДЫ}

В порядке проведения внутреннего контроля качества оказания медицинской помощи были произвольно выбраны 21 амбулаторная история болезни пациентов, находящихся в «листе ожидания» трансплантата печени в РНЦРХТ. Во всех представленных амбулаторных картах имелось заключение отборочной комиссии учреждения о наличии показаний к трансплантации печени и необходимости постановки пациента в ЛО. 11 пациентов являются жителями СанктПетербурга, 10 пациентов проживают в иных субъектах Северо-Западного федерального округа РФ. Группы сравнения были сформирова- ны по месту проживания пациентов: группа 1 жители СПб; группа 2 - иногородние пациенты. Мы не рассматривали отдельно ведение пациентов с различными диагнозами, приведшими к развитию печеночной недостаточности, так как в основу МЭС «Амбулаторное ведение пациентов в «листе ожидания» трансплантата печени» легло состояние (печеночная недостаточность), которое отмечалось у всех пациентов и явилось основанием для включения данных пациентов в «лист ожидания». Срок наблюдения исполнения МЭС составил 12 месяцев с момента включения пациентов в ЛО (конец 2013 - начало 2014 года), все пациенты остались в листе ожидания по истечению этого периода.

В качестве формализованного критерия проверки соблюдения МЭС использовалось среднее значение числа оказываемых услуг, приходящееся на одного пациента в массиве. Выборочные средние по отдельным услугам были сопоставлены с их средними значениями по стандарту.

Выполнение каждой услуги мы рассчитывали по формуле: $\lambda=$ ЧП×СК, которая выражает среднее число случаев оказания данной услуги, приходящееся на одного пациента в массиве.

Суммарное количество услуг в каждом разделе рассчитывали по формуле:

$\sum$ услуг $=($ СК $1 \times Ч П 1)+($ СК $2 \times Ч П 2)+\ldots+$ (СКn×ЧПn),

где $1, \ldots, \mathrm{n}$ - количество пациентов (амбулаторных карт) в выборке.

Реальное выполнение консультаций врачей

Таблица 1.

\begin{tabular}{lccc}
\hline & $\begin{array}{c}\text { Требование } \\
\text { М.Э.С, услуг }\end{array}$ & Группа 1, услуг & Группа 2, услуг \\
\hline \hline $\begin{array}{l}\text { Услуги врачей на 1 случай МП, } \\
\text { в соответствии с МЭС, 12 мес. }\end{array}$ & 29,4 & 4,6 & 1,5 \\
\hline Процент выполнения МЭС & 100 & 15,6 & 5,1 \\
\hline
\end{tabular}

Врачебные услуги, по которым МЭС может считаться исполненным

Таблица 2.

В01.047.01 Прием (осмотр, консультация) врача-терапевта первичный

B01.047.02 Прием (осмотр, консультация) врача-терапевта повторный

\begin{tabular}{cl}
\hline A25.14.001 & $\begin{array}{l}\text { Назначение лекарственной терапии при заболеваниях печени } \\
\text { и желчевыводящих путей }\end{array}$ \\
\hline A25.14.002 & $\begin{array}{l}\text { Назначение диетической терапии при заболеваниях печени } \\
\text { и желчевыводящих путей }\end{array}$ \\
\hline \multirow{2}{*}{25.14 .003} & $\begin{array}{l}\text { Назначение лечебно-оздоровительного режима при за- } \\
\text { болеваниях печени и желчевыводящих путей }\end{array}$ \\
\hline
\end{tabular}


Анализ оказания услуг рассмотрены по следующим сегментам медицинской помощи (МП): врачебные услуги, лабораторные услуги, инструментальные исследования.

\section{РЕЗУЛЬТАТЫ И ОБСУЖДЕНИЕ}

Выполнение врачебных услуг представлено в таблицах 1 и 2.

При этом следует отметить, что пациенты из группы 2 реже осматривались врачомтерапевтом, что объясняется их проживанием вдали от Центра, и, соответственно, более редкими посещениями врача-терапевта. В этой группе выполнение данной услуги составило 37,5\%.

Выполнение услуг консультаций врачейспециалистов было значимо ниже в обеих группах по сравнению с требованиями стандарта. Так осмотр врача-хирурга был выполнен только 24,3\% пациентов в группе 1 и 16,7\% пациентов в группе 2, при этом во второй группе 16,7\% нуждались в повторном осмотре. Частота осмотра неврологом составила соответственно 37,5 и 33,3\%. Медицинским психологом были осмотрены 22,5\% пациентов из группы 1 и 25\% пациен- тов из группы 2, при этом в повторной консультации медицинского психолога нуждались только пациенты группы 2 - 16,7\%. В таком /этом/ случае можно предположить недостаточное отражение данной медицинской услуги в первичной документации. Консультация эндокринолога была выполнена у 15\% пациентов из 1 группы, что может быть объяснено особенностями выбранных пациентов. Нельзя исключить, что иногородние пациенты получали данную услугу по месту жительства и это не нашло соответствующего отражения в амбулаторной карте Центра.

Выполнение лабораторных услуг представлено в таблицах 3 и 4.

Следует отметить, что исполнение стандарта в сегменте лабораторных услуг было достигнуто в основном за счет пациентов группы 2 . Это объясняется тем, что не все необходимые лабораторные исследования могут быть выполнены по месту жительства пациентов, и они их выполняют во время визитов в Центр. Исполнение остальных лабораторных услуг не превышает 45\%. Как показывает практика, лабораторные исследования выполняются по показани-

Реальное выполнение лабораторных услуг

Таблица 3.

\begin{tabular}{lccc}
\hline & $\begin{array}{c}\text { Требование } \\
\text { М.Э.С, услуг }\end{array}$ & Группа 1, услуг & Группа 2, услуг \\
\hline $\begin{array}{l}\text { Лабораторные услуги на 1 случай МП, } \\
\text { в соответствии с МЭС, 12 мес. }\end{array}$ & 333,2 & 124,3 & 133,4 \\
\hline \begin{tabular}{l} 
Процент выполнения МЭС \\
\hline
\end{tabular} & 100 & 37,3 & 40,03 \\
\hline
\end{tabular}

Лабораторные услуги, по которым МЭС может считаться исполненным

Таблица 4.

Общий (клинический) анализ крови развернутый

\begin{tabular}{ll}
\hline В03.016.03 & Общий (клинический) анализ крови развернутый \\
\hline В03.016.06 & Анализ мочи общий \\
\hline А09.05.009 & Исследование уровня С-реактивного белка в крови \\
\hline А09.05.016 & Исследование уровня аммиака в крови \\
\hline А09.05.018 & Исследование уровня мочевой кислоты в крови \\
\hline А09.05.043 & Исследование уровня креатинкиназы в крови \\
\hline А09.05.045 & Исследование уровня амилазы в крови \\
\hline А09.05.061 & Исследование уровня общего тироксина сыворотки (Т-4) крови \\
\hline А09.05.065 & Исследование уровня тиреотропина плазмы крови \\
\hline А09.05.121 & Исследование уровня тиреоглобулина в крови \\
\hline А09.05.200 & Молекулярно-биологическое исследование крови на онкомаркеры \\
\hline
\end{tabular}


ям не при каждом визите больного, что может служить основанием для изменения требований МЭС. Кроме того, не все результаты исследований, выполненных вне Центра, представлены в амбулаторных картах.

Лабораторные услуги у пациентов с недостаточной функцией печени целесообразно выполнять в специализированном лечебном учреждении, в «листе ожидания» которого они находятся. Такая организация процесса позволит: избегать неправильной оценки и трактовки динамики лабораторных показателей, своевременно выявлять показания к изменению терапии и, при необходимости, к стационарному лечению. Кроме того, тщательный контроль лабораторных показателей позволит выполнять пересадку печени нуждающемуся пациенту в «нужное время», что в свою очередь улучшит результаты дорогостоящего оперативного лечения (табл. 5.).

Данные услуги требуют серьезной коррекции СК и ЧП, вплоть до исключения из стандарта (табл. 6.).

Выполнение стандарта в разделе инструментальных исследований не превышает 55,6\% в отдельных видах исследований, таких как А04.06.001 «Ультразвуковое исследование селезенки», А04.14.001 «Ультразвуковое исследование печени», А04.14.002 «Ультразвуковое исследование желчного пузыря», А04.15.001 «Ультразвуковое исследование поджелудочной железы», А04.22.002 «Ультразвуковое исследование надпочечников», А04.28.001 «Ультразвуковое исследование почек». Все перечисленные услуги относятся к сонографии внутренних органов. По-видимому, в стандарт следует ввести сложную услугу - сонографию внутренних органов (табл. 7.).
Причиной недостаточного выполнения практически всех исследований в данном разделе МЭС послужили проблемы организации выполнения данных исследований в учреждении. В настоящее время они решены, следует ожидать более полного исполнения измененного стандарта.

Исполнение МЭС «Амбулаторное ведение пациентов в «листе ожидания» трансплантата печени» в РНЦРХТ может быть признано неудовлетворительным. Путём оптимизации исполнения стандарта послужат организационные и структурные изменения. В исполняющем учреждении в процесс приема и обследования пациентов, находящихся в ЛО, уже внесены организационные изменения. Представляется целесообразным изменить СК и ЧП в сторону уменьшения. При составлении МЭС авторы руководствовались рекомендациями Порядка оказания медицинской помощи по профилю «хирургия (трансплантация органов и (или) тканей человека)» (утв. приказом Министерства здравоохранения РФ от 31 октября 2012 г. N 567н, Зарегистрирован в Минюсте РФ 21 декабря 2012 г. Регистрационный N 26306), который предусматривает ежемесячное обследование пациентов, находящихся в «листе ожидания». Результаты трехлетнего исполнения подобного стандарта в Центре, выполняющем более 20 пересадок печени в год, имеющем ЛО в количестве 80 потенциальных реципиентов, выявили некоторые дефекты, допущенные при определении частоты и кратности исполнения медицинских услуг, включенных в стандарт. В связи с этим был проведен корреляционный анализ показателей услуг $\lambda$, предусмотренных МЭС и реально вы-

Не исполненные услуги (отсутствие данных в амбулаторных картах)

Таблица 5.

\begin{tabular}{cl}
\hline B03.005.06 & Коагулограмма (ориентировочное исследование системы гемостаза) \\
\hline B03.016.12* & Определение кислотно-основного соотношения \\
\hline A12.05.010 & НLA-антигены \\
\hline A12.05.055 & Время свертывания плазмы, активированное каолином \\
\hline
\end{tabular}

Таблица 6.

Реальное выполнение инструментальных исследований

\begin{tabular}{lccc}
\hline & $\begin{array}{c}\text { Требование } \\
\text { М.Э.С, услуг }\end{array}$ & Группа 1, услуг & Группа 2, услуг \\
\hline \hline $\begin{array}{l}\text { Инструментальные услуги на 1 случай МП, } \\
\text { в соответствии с МЭС, 12 мес. }\end{array}$ & 49,2 & 10,5 & 15,9 \\
\hline \begin{tabular}{l} 
Процент выполнения МЭС \\
\hline
\end{tabular} & 100 & 21,3 & 32,3 \\
\hline
\end{tabular}


Не исполненные инструментальные исследования

А04.10.002 Эхокардиография

А04.12.002 Ультразвуковая допплерография вен

А04.20.001 Ультразвуковое исследование матки и придатков

А04.21.001 Ультразвуковое исследование простаты

А06.20.004.007* Компьютерная томография органов малого таза у женщин с контрастированием с описанием и интерпретацией

A06.21.003.007* Компьютерная томография органов малого таза у мужчин с контрастированием с описанием и интерпретацией

полненных в течение 12 месяцев. В разделе врачебных услуг коэффициент корреляции составил 0,97 , в разделе лабораторных услуг - 0,97, в разделе инструментальных исследований - 0,96 в группе 1 и, соответственно, 0,96, 0,92 и 0,92 в группе 2.

\section{В Ы В 0 д Ы}

Полученные значения подтверждают предположение о том, что количества реально выполняемых услуг $\lambda$ достаточно для поддержания здоровья потенциального реципиента печеночного трансплантата на приемлемом уровне. Тем самым, мы подтвердили, что сокращение СК и

ЧП в рассматриваемом стандарте не повлечет ухудшение качества медицинской помощи пациентам, находящимся в गо. Подобное сокращение частоты и кратности услуг сделает стандарт более сбалансированным и удобным для исполнения.

Д.Н. Суслов ${ }^{1}$, А.В. Матвеева ${ }^{2}$ Н.Н. Рожков ${ }^{2}$ О.А. Герасимова ${ }^{1,3}$, Ф.К. Жеребцов

${ }^{1}$ ФББУ «Российский научный центр радиологии и хирургических технологий имени академика А.М. Гранова» Министерства здравоохранения Российской Федерации, Санкт-Петербург, Российская Федерация

${ }_{2}^{2}$ ФГБОУ ВО «Санкт-Петербургский государственный университет промышленных технологий и дизайна», СанктПетербург, Российская Федерация

${ }^{3}$ ФББОУ ВО «Санкт-Петербургский государственный университет", СанктПетербург, Российская Федерация.

\section{ОПТИМИЗАЦИЯ НАБЛЮДЕНИЯ И ЛЕЧЕНИЯ ПА- ЦИЕНТОВ В «ЛИСТЕ ОЖИДАНИЯ» ПЕЧЕНОЧНОГО ТРАНСПЛАНТАТА. ОПЫТ РЕГИОНАЛЬНОГО ЦЕНТРА}

Рассмотрены вопросы выполнения требований медико-экономического стандарта при оказании медицинскихуслугпациентам, ожидающимпересадку печени по данным одного лечебного учреждения. Анализ оказания услуг проведен по следующим сегментам: врачебные услуги, лабораторные услуги, инструментальные исследования. Выявлено, что реальное выполнение услуг в данных сегментах составило в группе жителей Санкт-Петербурга 15,6\%, 37,3\%, 21,3\% соответственно. В группе жителей других субъектов РФ эти показатели соответственно составили 5,1\%,40,03\% и 32,3\%.При этом,коэффициент корреляции услуг $\lambda$, предусмотренных стандартом и реально выполненных составил: в разделе врачебных услуг - 0,97, в разделе лабораторных услуг - 0,97, в разделе инструментальных исследований - 0,96 в группе жителей СПб и, соответственно, 0,96, 0,92 и 0,92 в группе жителей других субъектов РФ. Определены факторы, оказывающие влияние на выполнение требований медико-экономического стандарта. Полученные результаты подтверждают предположение о том, что количества реально выполняемых услуг $\lambda$ достаточно для поддержания здоровья потенциального реципиента печеночного трансплантата на приемлемом уровне. Сокращение кратности и частоты предоставления услуг в рассматриваемом стандарте не повлечет ухудшение качества медицинской помощи пациентам, находящимся в «листе ожидания», но сделает стандарт более сбалансированным и удобным для исполнения.

Ключевые слова: медико-экономический стандарт, медицинские услуги, «лист ожидания», пересадка печени. 


\section{D.N. Suslov ${ }^{1}$, A.V. Matveeva ${ }^{2}$, N.N. Rozhkov ${ }^{2}$, O.A. Gerasimova' ${ }^{1,3}$, F.K. Zherebtsov ${ }^{1}$}

${ }^{1}$ A.M. Granov Russian scientific center of radiology and surgery technologies, Saint Petersburg, Russian Federation ${ }^{2}$ Saint Petersburg State University of Industrial Technologies and Design, Saint Petersburg, Russian Federation

${ }^{3}$ Saint Petersburg State University, Saint Petersburg, Russian Federation

\section{OBSERVATION AND TREATMENT OF PATIENTS IN THE LIVER TRANSPLANT «WAITING LIST» OPTIMIZATION. THE REGIONAL CENTER EXPERIENCE}

Discussed issues of implementation of the requirements of medico-economic standard in providing medical services to patients awaiting liver transplantation according to one medical institution. Analysis services conducted in the following segments: medical services, laboratory services, instrumental studies. It is revealed that the real performance of the services in these segments were in the group of inhabitants of St.-Petersburg of $15.6 \%$ and $37.3 \%, 21.3 \%$, respectively. In the group of residents of other subjects of the Russian Federation, these figures respectively amounted to $5.1 \%, 40.03 \%$ and $32.3 \%$. In this case, the correlation coefficient $\lambda$ of services specified by the standard and really carried: in the section for physician services are 0.97 , see the laboratory services are 0.97 , see research tools -0.96 volts to a group of residents of St. Petersburg and, accordingly, of 0.96 , and 0.920 .92 in the residents of other subjects of the Russian Federation. Was defined the factors influencing the implementation of the requirements of medicoeconomic standard. The obtained results confirm the assumption that the number of actually performed services of $\lambda$ is sufficient to maintain the potential recipient of a liver transplant status at an acceptable level. The reduction of the multiplicity and frequency of service provision in the standard will not cause deterioration in the quality of medical care to patients in the «waiting list» but will done the standard more balanced and comfortable for performance.

Key words: medico-economic standard, health care, «waiting list», liver transplantation.

\section{ЛИТЕРАТУРА}

1. Суслов Д.Н., Рожков Н.Н., Матвеева А.В. Идеи совершенствования структуры медико-экономических стандартов с применением математического моделирования. Общественное здоровье и здравоохранение. 2016; 3: 26-30.

2. Rosen H.R., Shackleton C.R., Martin P.: Indications for and timing of liver transplantation. Med Clin North Am 1996; 80: 1069-1102. PMID: 8804375

3. Хубутия М.Ш., Андрейцева О.И., Журавель С.В., Гуляев В.А., Салиенко В.А., Чжао А.В. Методика формирования и ведения «листа ожидания» трансплантации печени. Трансплантология 2009; 1: 13-19.

4. Таразов П.Г., Гранов Д.А., Поликарпов А.А., Генералов М.И., Жеребцов Ф.К., Кротова О.А. и др. Ортотопическая трансплантация печени: роль интервенционной радиологии. Вестник трансплантологии и искусственных органов. 2009;11(3):42-50. DOI:10.15825/19951191-2009-3-42-50

5. Михайличенко Т.Г., Буравко К.А., Щелкова О.Ю. Эмоционально-личностный статус больных вирусными гепатитами в период ожидания трансплантации печени. Медицинская психология в России. 2016; 37 (2): URL: http://mprj.ru

\section{REFERENCES}

1. Suslov D.N., Rozhkov N.N., Matveeva A.V. Ways to improve the structure of medical and economic standards based on mathematical modeling. 2016; 3: 26-30 (in Russian).

2. Rosen H.R., Shackleton C.R., Martin P.: Indications for and timing of liver transplantation. Med Clin North Am 1996; 80: 1069-1102. PMID: 8804375

3. Khubutia M.Sh., Andreitseva O.I., Zhuravel S.V., Gulyaev V.A., Saliyenko A.A., Chzhao A.V. Procedure for drawing up and keeping a liver transplantation waiting list. Transplantology 2009; 1: 13-19 (in Russian).

4. Tarazov P.G., Granov D.A., Polikarpov A.A., Generalov M.I., Zherebtsov F.K., Krotova O.A. et al. Orthotopic liver transplantation: role of interventional radiology. Russian Journal of Transplantology and Artificial Organs. 2009;11(3):42-50 (in Russian). DOI:10.15825/1995-11912009-3-42-50

5. Mikhaylichenko T.G., Buravko K.A., Shchelkova O.Yu. Emotsional'no-lichnostnyystatusbol'nykhvirusnymigepat itamivperiodozhidaniyatransplantatsiipecheni. Meditsinskayapsikhologiya v Rossii. 2016; 37 (2): URL: http://mprj. ru (in Russian). 